\title{
Editorial: Attention and Methylphenidate
}

\author{
Avi Avital ${ }^{1 *}$, Iris Manor $^{2}$ and David Coghill ${ }^{3,4}$ \\ ${ }^{1}$ Department of Neuroscience, Rappaport Faculty of Medicine, Emek Medical Center, Technion - Israel Institute of \\ Technology, Haifa, Israel, ${ }^{2}$ ADHD Clinic, Geha MHC and Clalit, Sackler School of Medicine, Tel Aviv, Israel, ${ }^{3}$ Department of \\ Paediatrics, Faculty of Medicine, Dentistry and Health Sciences, University of Melbourne, Melbourne, VIC, Australia, \\ ${ }^{4}$ Department of Psychiatry, Faculty of Medicine, Dentistry and Health Sciences, University of Melbourne, Melbourne, VIC, \\ Australia
}

Keywords: ADHD, diagnosis, methylphenidate, adherence, anxiety, dysregulation, dose, non-responder

\section{Editorial on the Research Topic}

\section{Attention and Methylphenidate}

Attention Deficit Hyperactivit Disorder, more simply known as ADHD, is a neurodevelopmental disorder characterized by inattention, hyperactivity, and impulsivity. There are three major presentations: primarily hyperactive and impulsive, primarily inattentive, and combined type (Gaub and Carlson, 1997). However, despite the differences between the subtypes, methylphenidate (MPH), a psychostimulant, is the primary pharmaceutical treatment of choice (Heal et al., 2009).

While MPH is largely an effective treatment across the subtypes (O'Driscoll et al., 2005), there is a large issue with adherence to treatment. In her contribution, Kritchman et al. findings suggested that acute MPH administration induces a delayed anxiogenic response. Considering ADHD-anxiety comorbidity, acute doses of MPH can induce anxiety, while, paradoxically, chronic MPH treatment has anxiolytic effects (Zubedat et al., 2019). There can be large difference in dose-response to $\mathrm{MPH}$, even when a patient adheres to ADHD treatment. Additionally, some ADHD patients do not respond to medication at all, despite displaying typical ADHD symptoms. These "non-responders" may decrease adherence to MPH treatment, because they perceive the medication to be ineffective. The exact cause for non-responders is unknown, and it may stem from a puzzling, non-linear dose response to $\mathrm{MPH}$.

The subset of ADHD non-responders may be mitigated with improved objective diagnostic assessments of ADHD. Throughout one's life, a variety of circumstances influence the individual's attention. For instance, high levels of stress, exhaustion and hunger, can all have large implications on attention, and are common dysregulating experiences throughout one's life. Since there are no sufficiently objective and accurate diagnostics for ADHD, subjects experiencing temporary inattention due to external circumstances may be misdiagnosed. These misdiagnoses could account for the number of non-responders to MPH treatment, as these "non-responders" could simply be patients suffering from transient inattention, rather than a true neurodevelopmental disorder. In the past 25 years the rates of ADHD diagnosis have increased (Mahone and Denckla, 2017), and while this may be due to a true increase in ADHD rates, lack of objective diagnostic protocols can increase misdiagnosis.

While these disparities in dose-response are puzzling, many techniques may shed light on these issues. In her contribution, Wigal et al. described the Laboratory School Protocol (LSP), 
a research protocol in which students are placed in a classroom environment where they are medicated and their behavior is evaluated. In her contribution, Rinker et al. postulated that ADHD affects academic functioning regardless of education level; Thus, LSPs are applicable to ADHD regardless of education level. In LSPs, medication is given at the research site to control for temporal differences in application, elucidating on the large variation in dose-response to MPH in ADHD patients. Wigal et al. reported that placebo effects are reduced when research environments mirror situations in which symptoms would be aggravated and when medication administration is tightly controlled, altogether aiding in the establishment of more objective ADHD diagnostic tests.

LSPs utilize the Permanent Product Measure of Performance Math Test (PERMP) as the most objective ADHD diagnostic test to date. However, promising research regarding norepinephrine receptors could lead to objective diagnosis even outside of simulated classroom environments like the LSP and exams like the PERMP. In their commentary on van den Brink et al. (2018), Medel et al. contributed that the differential expression of $\alpha 1$ and $\alpha 2$ norepinephrine (NE) receptors can induce heterogeneous spatial modes, a shifting of "brain states." Thus, suggesting that there is a dynamic shaping of brain activity by neuromodulations through the heterogeneous expression of $\alpha 1$ and $\alpha 2$ NE receptors that creates spontaneous fluctuations in cognition, elucidating on data previously established by Shine et al. (2019). In her contributed mini-review, Wigal et al. further supports this suggestion by claiming that ADHD itself is not necessarily an attention deficit, but rather inconsistent and misdirected attention. Likewise, according to Spiegel and Pollak contributed research, ADHD encompasses a lack of proper evaluation of risk-taking behavior. Hypothetically, those with ADHD do not necessarily have limited attention, but the attention is misdirected through varying brain states due to differential expression of $\alpha 1$ and $\alpha 2$ NE receptors. Practically, Medel et al. show that pupillometry can measure varying levels of NE with different brain states, establishing a possible objective diagnostic measure of ADHD. Together, pupillometry and the PERPM may prove to be a robust, objective diagnostic measure of $\mathrm{ADHD}$, which may limit misdiagnosis in $\mathrm{ADHD}$ and the number of "non-responders" to ADHD treatment.

While MPH and behavioral therapies are available, there are prevention techniques which may help to reduce the

\section{REFERENCES}

Gaub, M., and Carlson, C. L. (1997). Behavioral characteristics of DSM-IV ADHD subtypes in a school-based population. J. Abnormal Child Psychol. 25, 103-111. doi: 10.1023/A:1025775311259

Heal, D. J., Cheetham, S. C., and Smith, S. L. (2009). The neuropharmacology of ADHD drugs in vivo: insights on efficacy and safety. Neuropharmacology 57, 608-618. doi: 10.1016/j.neuropharm.2009.08.020

Linssen, A. M. W., Sambeth, A., Vuurman, E. F. P. M., and Riedel, W. J. (2014). Cognitive effects of methylphenidate in healthy volunteers: a review of single dose studies. Int. J. Neuropsychopharmacol. 17, 961-977. doi: $10.1017 /$ S1461145713001594 development of ADHD altogether. Tapping the black box of $\mathrm{ADHD}$ on its translational face, previous reports utilizing animal models showed that the application of $\mathrm{MPH}$ alone and/or with enriched environment (EE) on adult offspring rescues the detrimental effects of prenatal stress attentional reactivity (Zubedat et al., 2015). The influence of EE and MPH could be translated and confirmed by conducting LSP studies, hence, elucidating on ways EE may be increased in school systems to aid in ADHD symptoms.

Taken with these results, MPH has clinical applications outside of ADHD. Acute MPH improves cognitive ability in those with and without ADHD (Linssen et al., 2014). Temporary use of $\mathrm{MPH}$ to improve cognition may be appropriate in professions where inattention has true large implications in the livelihood of others (e.g., medical doctors on extended shift). However, as previously mentioned, side effects such as temporarily increased anxiety must be taken into account when $\mathrm{MPH}$ is acutely administered.

In essence, adherence issues with $\mathrm{MPH}$ treatment in those with ADHD may be rooted in acute increases in anxiety, non-responders, and misdiagnosis. These adherence issues could be mediated by assuring patients anxiogenic effects are temporary. In order to accurately diagnose patients with ADHD, we must focus on discovering and implementing objective diagnostic measures such as pupillometry. In order to decrease ADHD diagnosis overall, we can increase research on potential prevention methods such as environmental enrichment, as well as on the effects of adverse life events, such as prenatal stress, on attention.

\section{AUTHOR CONTRIBUTIONS}

AA conceptualized and wrote the article. IM and DC consulted and edited the paper.

\section{ACKNOWLEDGMENTS}

The authors would like to thank all the investigators who contributed articles to this Research Topic. The authors would like to thank Ms. Anna Grossman for her assistance in preparing this editorial. Phenylbutazone (PBZ) and historical prospective. J. Int. Neuropsychol. Soc. 23, 916-929. doi: 10.1017/S1355617717000807

O'Driscoll, G. A., Dépatie, L., Holahan, A. L. V., Savion-Lemieux, T., Barr, R. G., Jolicoeur, C., et al. (2005). Executive functions and methylphenidate response in subtypes of attention-deficit/hyperactivity disorder. Biol. Psychiatry 57, 1452-1460. doi: 10.1016/j.biopsych.2005. 02.029

Shine, J. M., Breakspear, M., Bell, P. T., Martens, K. E., Shine, R., Koyejo, O., et al. (2019). Human cognition involves the dynamic integration of neural activity and neuromodulatory systems. Nat. Neurosci. 22, 289-296. doi: 10.1038/s41593-018-0312-0 
van den Brink, R. L., Nieuwenhuis, S., and Donner, T. H. (2018). "Amplification and suppression of distinctbrainwide activity patterns by catecholamines." J. Neurosci. 38, 7476-7491. doi: 10.1523/JNEUROSCI.0 514-18

Zubedat, S., Aga-Mizrachi, S., Cymerblit-Sabba, A., Ritter, A., Nachmani, M., and Avital, A. (2015). Methylphenidate and environmental enrichment ameliorate the deleterious effects of prenatal stress on attention functioning. Stress 18, 280-288. doi: 10.3109/10253890.2015.1 023790

Zubedat, S., Havkin, E., Maoz, I., Aga-Mizrachi, S., and Avital, A. (2019). A probabilistic model of startle response reveals opposite effects of acute versus chronic methylphenidate treatment. J. Neurosi. Methods 327:108389. doi: 10.1016/j.jneumeth.2019. 108389
Conflict of Interest: Independent of this editorial DC has received research funding from Takeda/Shire, honoraria from Takeda/Shire, Medice and Servier.

The remaining authors declare that the research was conducted in the absence of any commercial or financial relationships that could be construed as a potential conflict of interest.

Copyright (C) 2020 Avital, Manor and Coghill. This is an open-access article distributed under the terms of the Creative Commons Attribution License (CC BY). The use, distribution or reproduction in other forums is permitted, provided the original author(s) and the copyright owner(s) are credited and that the original publication in this journal is cited, in accordance with accepted academic practice. No use, distribution or reproduction is permitted which does not comply with these terms. 